\title{
Erratum to: Update of Conservative Systemic Treatment of Uterine Fibroids
}

\author{
Magdalena Maria Zalewski • Felix Zeppernick • \\ Joseph Neulen
}

Published online: 10 August 2014

(C) Springer Science+Business Media New York 2014

\section{Erratum to: Curr Obstet Gynecol Rep DOI 10.1007/s13669-014-0089-4}

\begin{abstract}
Uterine fibroids are a common disease in women and lead to different symptoms, like pain and bleeding disorders. Apart from surgical treatment, there are many medical treatment options, which are presented in this article. Combined oral contraceptives and Levonorgestrel-releasing intrauterine systems are possible options for bleeding disorders that are a consequence of uterine fibroids. Gonadotropinreleasing hormone agonists and the new selective progesterone receptor modulator ulipristal acetate can effectively reduce myoma mass and vaginal bleeding rate. Ulipristal acetate should especially be considered for treatment in symptomatic women, as it has only a few noteworthy side effects.
\end{abstract}

Due to errors introduced during review process, we are republishing the entire article in its corrected format including the name of Dr. F.

Zeppernick as second author. The online version of the original article can be found at http://dx.doi.org/10.1007/s13669-014-0089-4.

M. M. Zalewski $(\bowtie) \cdot$ F. Zeppernick

Klinik für Gynäkologie und Geburtsmedizin, Uniklinik RWTH

Aachen, Pauwelsstraße 30, 52074 Aachen, Germany

e-mail: mzalewski@ukaachen.de

F. Zeppernick

e-mail: fzeppernick@ukaachen.de

F. Zeppernick

Department of Pathology, Johns Hopkins University

School of Medicine, Baltimore, MD 21231, USA

J. Neulen

Klinik für Gynäkologische Endokrinologie und

Reproduktionsmedizin, Uniklinik RWTH Aachen, Pauwelsstraße

30, 52074 Aachen, Germany

e-mail:jneulen@ukaachen.de
Keywords Uterine fibroids · Progesterone $\cdot$ Combined oral contraceptives $\cdot$ Levonorgestrel-releasing intrauterine systems · Gonadotropin-releasing hormone agonists . Ulipristal acetate

\section{Introduction}

Uterine fibroids or uterine leiomyomas are the most common pelvic tumors in women. Mostly, they are benign tumors and arise from smooth muscle cells of the myometrium in women of reproductive age.

While many fibroids are asymptomatic, some patients suffer from a variety of symptoms. Most symptoms can be classified into three main categories: 1) pelvic pressure and pain; 2) symptoms of abnormal uterine bleeding, such as heavy and /or prolonged bleeding; and 3) reproductive dysfunctions that encompass infertility issues as well as adverse pregnancy outcomes.

The variety of symptoms reflects the fact that individualized treatment concepts are necessary. Apart from the more invasive and definite surgical and interventional therapy options such as myomectomy, hysterectomy and uterine artery embolization that are presented in this issue, several medical treatment measures should be taken into consideration and discussed with the patient. An overview of medical treatment options and their side effects will be presented here.

Pathogenesis of Uterine Fibroids

To understand the medical treatment options, it is helpful to briefly review the pathogenesis of uterine fibroids, especially their hormone-dependent growth characteristics (an extensive overview on the pathogenesis and the genetic background of uterine fibroids can be found in this issue).

Although the pathogenesis of fibroids is complex and not completely understood, it appears that the development of uterine fibroids depends on steroid hormones. Traditionally, estrogens 
were thought to be the most important factor in the growth of leiomyomas. There was strong evidence for this theory in vitro [1]. In contrast, the role of progesterone remained unclear after in vitro studies. Using a xenograft model, the effects of steroid hormones on fibroids were explored in a mouse model [2]. It was shown that the treatment with estrogen alone did not induce growth of fibroids. But estrogen led to an increased expression of progesterone receptor, which resulted in a progesterone dependent growth of fibroids. Taken together, these results suggest that the development of uterine fibroids is dependent on the interaction of both of these steroid hormones. Here, sensitivity to and proliferative effects of progesterone are prominent during fibroid proliferation. This knowledge helps us to understand the different therapeutic options.

\section{Combined Oral Contraceptives}

The majority of oral contraceptives are combined estrogenprogestin contraceptives. Apart from their birth control effects, they show several noncontraceptive benefits that are commonly used in gynecological practice. In many cases, this reflects a symptomatic approach rather than a causal therapy.

Combined oral contraceptives can be useful in patients with hypermenorrhea as the primary symptom of uterine fibroids, especially when they are used in extended cycles. By taking these hormonal contraceptives, regulation of the heavy menstrual bleeding can be achieved [3]. But these contraceptives do not seem to reduce the volume of the fibroids [4], even though there is some evidence that the risk of developing uterine fibroids during the intake of combined contraceptives is actually reduced [5].

Opinions vary regarding the effects of oral contraceptives on the volume of fibroids. Some studies even suggest that oral contraceptives can increase the volume of leiomyomas [6].

In summary, combined oral contraceptives are a possible treatment option for bleeding disorders due to uterine fibromas, but not for the fibromas themselves.

\section{Levonorgestrel-Releasing Intrauterine System (LNG-IUS)}

The Levonorgestrel-releasing intrauterine system was initially indicated for intrauterine contraception for 5 years (http://www. accessdata.fda.gov/drugsatfda_docs/label/2008/021225s019lbl. pdf). In 2009, the U.S. Food and Drug Administration (FDA) approved additional use for the LNG-IUS to treat heavy menstrual bleeding (http://www.fda.gov/newsevents/newsroom/ pressannouncements/2009/ucm184747.htm). Although there are no randomized trials directly evaluating LNG-IUS use for fibroid-related menorrhagia, there is evidence that LNG-IUS is effective in the treatment of heavy menstrual bleeding, independent of the reason for the bleeding [7, 8]. Compared to a surgical treatment via hysterectomy, the patients were as satisfied and had a similar quality of life as after surgery, whereas the costs were $40 \%$ lower in the LNG-IUS-treated group.

A systematic review could showed that a leiomyomaassociated hypermenorrhea can be efficiently reduced by this method. Additionally, women showed higher serum levels of hemoglobin, hematocrit and ferritin after beginning the therapy [9]. Even compared to the treatment with combined oral contraceptives, the LNG-IUS was more effective in reducing heavy menstrual bleeding in women with fibroid-related menorrhagia [10]. These data are surprising, because progesterone should be a growth factor for uterine fibroids. It is assumed that the regulation of heavy menstrual bleeding is a result of the inhibition of endometrial proliferation by progesterone [1].

However, there seems to be no significant reduction of the volume of uterine fibroids after treatment with LNG-IUS [11]. Only a few trials with small numbers of patients showed a decrease in the myoma mass [12].

Considering the limited side effects of the LNG-IUS treatment, there is good reason to attempt this therapy before performing surgery. But still there are adverse events to be considered. The most common one associated with the LNGIUS is menstrual bleeding irregularity [13]. After 12 months of use, $20 \%$ of the women are amenorrheic, and up to $70 \%$ of users are oligomenorrheic or amenorrheic after 24 months. For some women this might be an acceptable or even positive side effect, but others cannot tolerate this irregularity and want the device removed [14]. The former worries about a higher rate of pelvic inflammatory disease (PID) in patients with LNG-IUS cannot be clearly verified [15]. The higher rate of PID rather seems to be related to the tails that used to be braided and could potentially have allowed ascending bacteriosis. Modern intrauterine devices (IUDs) have monofilament tail strings that have not been associated with increased risk of infections - apart from the risk associated with insertion. There is also a small rate of patients with spotting or intermenstrual bleeding, ovarian cysts, acne, weight gain, depression and decreased libido. Nevertheless, a study that investigated long-term acceptability of an LNG-IUS showed that it is a well-accepted treatment [16]. The decrease of dysmenorrhea was especially evaluated as a very positive consequence. In another study, $84 \%$ of the patients reported improvement of menstrual discomfort. [17]

Possible limitations to this therapy can be leiomyomas that cause distortion of the uterine cavity or some intracavitary leiomyomas that make the insertion impossible. Even after a successful insertion, the risk of expulsion is higher in patients with leiomyomas [18]. Expulsion rates in these patients are reported to be between $0 \%$ and $20 \%$, whereas in patients without leiomyomas they are normally between $0 \%$ and $3 \%$. A thorough ultrasound evaluation before and after insertion can help to prevent expulsion related problems.

The data implies that LNG-IUS are a well-accepted, symptom-oriented treatment option for some patients with uterine fibroids, especially if hypermenorrhea is present. 


\section{Progestin-Only Oral Contraception}

Although the substances in progestin-only oral contraceptives are similar to the LNG-IUS, this treatment is no standard for uterine fibroids. There is no documented effect on the volume of fibroids [19]. Furthermore, the increased rate of side effects and the lower Pearl-Index should be considered before choosing progestin-only oral contraceptives for treatment of fibroid related symptoms.

Gonadotropin-Releasing Hormone Agonist (GnRH Agonist)

Gonadotropin-releasing hormone agonists are treatment options, not just for control of symptoms for women with uterine fibroids. These medications are able to reduce the release of sexual steroids via their influence on the pituitary gland [20]. Treatment with GnRH agonists initially produces an increase in pituitary secretion of the gonadotropins LH and FSH, followed by a sustained downregulation with a decrease of secretion of LH and FSH, which will lead to a hypogonadotropic, hypogonadal state [21].

Consequently, levels of estradiol and progesterone decrease and the fibroids are deprived of their growth impulse. As a result, the volume of myomas can be effectively reduced by around $35-60 \%[22,23]$. In vitro data suggests additional direct effects of GnRH agonists on the fibroid and endometrial cells that cause apoptosis of these cells [24].

These mechanisms result in a decrease of fibroid-related symptoms, especially hypermenorrhea. Menstrual bleeding can be reduced with a consecutive normalization of the hemoglobin level [25]. In most cases, the application of GnRH agonists leads to amenorrhea.

However, after the end of the therapy with GnRH agonists, there is often a rebound effect in symptoms and fibroid regrowth [26]. Hence, treatment with GnRH agonists is only indicated for a timely, limited, preoperative therapy of uterine fibroids.

Patients should be carefully selected for this treatment. In an unselected cohort, there was no evidence of benefit for preoperative treatment, regarding the possible complications such as intraoperative blood loss, compared to an operation without previous treatment with GnRH agonist. [27, 28] There was also no evidence for a difference in postoperative morbidity and hospital stay.

Due to climacteric side effects such as hot flashes, sleep disorder, myalgia, vaginal dryness and mood swings, GnRH agonists have a variable agreeability [23, 29]. Furthermore, this treatment can lead to reduced bone mineral density and finally to osteoporosis with consecutive serious complications like dangerous bone fractures if applied for more than six months $[30,31]$. Therefore, an add-back therapy (with hormone replacement therapy) should be considered. A possible add-back treatment contains estrogen, at times combined with progesterone in low dose. Thereby side effects can be reduced without leading to a growth stimulus on the fibroids.

\section{Ulipristal Acetate (UPA)}

In recent years, a new treatment for uterine fibroids has been established. Ulipristal acetate is a selective progesterone receptor modulator (SPRM) blocking most progestational effects with reduced antiglucocorticoid activity [32]. UPA significantly reduces uterine fibroid volume, reduces menstrual blood loss, and improves quality of life without serious adverse events [33]. Recently, three large trials were published that analyzed the efficacy of UPA and resulted in the approval of this SPRM for clinical use.

The PEARL I trial compared a treatment of UPA against placebo treatment for menorrhagia. More than 240 women were included in the study and randomized to three groups. The medication was given orally for 13 weeks. The selected doses were $5 \mathrm{mg}$ for one group and $10 \mathrm{mg}$ for the other treatment group versus a placebo for a third group. The observed effect of UPA was a significant reduction of fibroid volume, as well as a significantly reduced vaginal bleeding rate [34]. Eighty percent of the treated women reported an improved bleeding profile. There was also a high rate of amenorrhoea (63\% in the $5 \mathrm{mg}$ group and $71 \%$ in the $10 \mathrm{mg}$ group), which was a satisfactory result for the patients. In comparison, the placebo group experienced regular menstrual bleeding with a similar bleeding intensity in $80 \%$. Some women still suffered irregular bleeding under the treatment, which especially occurred in patients with sub-mucous fibroids.

The PEARL II trial compared treatment with UPA in a dose of $5 \mathrm{mg}$ and $10 \mathrm{mg}$ with the former most effective medical therapy with monthly injections of leuprolin acetate in a noninferiority setting [35]. Three hundred and seven patients were included in this randomized double-blind trial. All three groups had very good bleeding control during treatment. In the UPA treated group the bleeding was attenuated more rapidly than in the GnRH agonist group. Patients in the UPA group noticed an improvement of the bleeding profile in $90 \%$ and $98 \%$, whereas the leuprolide group observed an improvement in $89 \%$. For all patients, a significant reduction of fibroid volume could be demonstrated; it was strongest in the leuprolide group. However, there was less rebound effect seen in the UPA groups. After UPA therapy, the reduction in size effect lasted longer than after the treatment with leuprolide. Comparing the side effects, UPA was more accepted. Rates of moderate to severe hot flashes were significantly less with UPA than with leuprolin acetate (10\% and $11 \%$ in UPA groups compared to $40 \%$ ). There was also good evidence for a higher rate of bone resorption, implicating a higher risk for osteoporosis after treatment with leuprolide. A common change seen after treatment with UPA is a change in endometrial thickness. This side effect could only be observed in UPA treatment groups and is not 
known for treatment with GnRH agonists. Endometrial biopsies, however, showed benign endometrial changes without any higher rate of premalignant lesions. Hence, there seems to be no clinical consequence of these so-called PAECs (ProgesteroneReceptor-Modulator associated endometrial changes) [36].

The recently published PEARL III trial investigated the efficacy and safety of long-term treatment with UPA [37]. In the former trials, UPA was used for only 13 weeks; therefore, there were no results for a longer treatment. In this trial, UPA was given for up to four courses of 13 weeks, with short off-treatment periods between each course including one menstrual bleeding and the beginning of a second. The whole treatment lasted 18 months. During this regime, UPA was able to induce high rates of amenorrhea in over $90 \%$ of women. Also, the efficacy in shrinking fibroids could be confirmed. The median volume reduction after four courses was $-72.1 \%$, whereas a 13 -week period led to a reduction of $-45.1 \%$. Regarding these results, UPA seems to be a good candidate for a long-term treatment, especially because there are no approved alternatives. By comparison GnRH agonists should be used carefully as there are severe side effects, even when the GnRH agonists are combined with an add-back therapy, and treatment should be limited in time.

In all three PEARL trials, no severe side effects of ulipristal acetate could be detected. The most often reported side effects were headaches, nasopharyngitis and abdominal pain.

In the European Union, ulipristal acetate is available for two courses of 3 months for the conservative treatment of uterine fibroids [38].

\section{Conclusion}

In summary, apart from surgical and interventional treatment options, several hormonal treatment options should be considered for women with uterine fibroids. Sexual steroids, i.e., contraceptives or LNG-IUS, can reduce symptoms conveyed by fibroma. GnRH agonists and ulipristal acetate are effective in systemic treatment, not only to reduce symptoms related to fibroma but also to reduce fibroma masses. Also, they might be considered as a preoperative treatment to reduce uterine fibroid size to facilitate subsequent surgery. This can lead to an easier operative approach. Also, the shrinkage of fibroids could reduce surgery-related morbidity and decline intraoperative blood loss. Today, UPA is the most effective medication for conservative treatment of uterine fibroma.

\section{Compliance with Ethics Guidelines}

Conflict of Interest All authors declare that they have no conflict of interest.

Human and Animal Rights and Informed Consent This article does not contain any studies with human or animal subjects performed by any of the authors.

\section{References}

1. Kim JJ, Kurita T, Bulun SE. Progesterone action in endometrial cancer, endometriosis, uterine fibroids, and breast cancer. Endocr Rev. 2013;34(1):130-62.

2. Ishikawa H, Ishi K, Serna VA, Kakazu R, Bulun SE, Kurita T. Progesterone is essential for maintenance and growth of uterine leiomyoma. Endocrinology. 2010;151:2433-42.

3. Sweet MG, Schmidt-Dalton TA, Weiss PM, Madsen KP. Evaluation and management of abnormal uterine bleeding in premenopausal women. Am Fam Physician. 2012;85(1):35-43.

4. Hoellen F, Griesinger G, Bohlmann MK. Therapeutic drugs in the treatment of symptomatic uterine fibroids. Expert Opin Pharmacother. 2013;14(15):2079-85.

5. Qin J, Yang T, Kong F, Zhou Q. Oral contraceptive use and uterine leiomyoma risk: a meta-analysis based on cohort and case-control studies. Arch Gynecol Obstet. 2013;288(1):139-48.

6. Wise LA, Palmer JR, Harlow BL, Spiegelman D, Stewart EA, Adams-Campbell LL, et al. Reproductive factors, hormonal contraception, and risk of uterine leiomyomata in African-American women: a prospective study. Am J Epidemiol. 2004;159(2):113-23.

7. Wildemeersch D, Andrade A. Review of clinical experience with the frameless LNG-IUS for contraception and treatment of heavy menstrual bleeding. Gynecol Endocrinol. 2010;26(5):383-9.

8. Heikinheimo O, Gemzell-Danielsson K. Emerging indications for the levonorgestrel-releasing intrauterine system (LNG-IUS). Acta Obstet Gynecol Scand. 2012;91(1):3-9.

9. Zapata LB, Whiteman MK, Tepper NK, Jamieson DJ, Marchbanks PA, Curtis KM. Intrauterine device use among women with uterine fibroids: a systematic review. Contraception. 2010;82(1):41-55.

10. Sayed GH, Zakherah MS, El-Nashar SA, Shaaban MM. A randomized clinical trial of a levonorgestrel-releasing intrauterine system and a low-dose combined oral contraceptive for fibroid-related menorrhagia. Int J Gynaecol Obstet. 2011;112(2):126-30.

11. Kriplani A, Awasthi D, Kulshrestha V, Agarwal N. Efficacy of the levonorgestrel-releasing intrauterine system in uterine leiomyoma. Int J Gynaecol Obstet. 2012;116(1):35-8.

12. Jindabanjerd K, Taneepanichskul S. The use of levonorgestrel-IUD in the treatment of uterine myoma in Thai women. J Med Assoc Thail. 2006;89 Suppl 4:S147-51.

13. Beatty MN, Blumenthal PD. The levonorgestrel-releasing intrauterine system: safety, efficacy, and patient acceptability. Ther Clin Risk Manag. 2009;5(3):561-74.

14. Cox M, Tripp J, Blacksell S. Clinical performance of the levonorgestrel intrauterine system in routine use by the UK Family Planning and Reproductive Health Research Network: 5-year report. J Fam Plann Reprod Health Care. 2002;28(2):73-7.

15. Alton TM, Brock GN, Yang D, Wilking DA, Hertweck SP, Loveless MB. Retrospective review of intrauterine device in adolescent and young women. J Pediatr Adolesc Gynecol. 2012;25(3):195-200.

16. Baldaszti E, Wimmer-Puchinger B, Löschke K. Acceptability of the long-term contraceptive levonorgestrel-releasing intrauterine system (Mirena): a 3-year follow-up study. Contraception. 2003;67(2):8791.

17. Radesic B, Sharma A. Levonorgestrel-releasing intrauterine system for treating menstrual disorders: a patient satisfaction questionnaire. Aust N Z J Obstet Gynaecol. 2004;44(3):247-51.

18. Kim ML, Seong SJ. Clinical applications of levonorgestrel-releasing intrauterine system to gynecologic diseases. Obstet Gynecol Sci. 2013;56(2):67-75.

19. Khan AT, Shehmar M, Gupta JK. Uterine fibroids: current perspectives. Int J Womens Health. 2014;6:95-114.

20. Kiesel L, Runnebaum B. Gonadotropin-releasing-Hormon und Analoga, Physiologie und Pharmakologie. Gynakol Geburtshilfliche Rundsch. 1992;32:22-30. 
21. Barbieri RL. Clinical applications of GnRH and its analogues. Trends Endocrinol Metab. 1992;3(1):30-4.

22. Minaguchi H, Wong JM, Snabes MC. Clinical use of nafarelin in the treatment of leiomyomas. A review of the literature. J Reprod Med. 2000;45(6):481-9.

23. Friedman AJ, Barbieri RL, Doubilet PM, Fine C, Schiff I. A randomized, double-blind trial of a gonadotropin releasing-hormone agonist (leuprolide) with or without medroxyprogesterone acetate in the treatment of leiomyomata uteri. Fertil Steril. 1988;49(3):404-9.

24. Kang J, YuWang D, XiaWang X. Yu J Up-regulation of apoptosis by gonadotrophin-releasing hormone agonist in cultures of endometrial cells from women with symptomatic myomas. Hum Reprod. 2010;25(9):2270-5.

25. Koskas M, Chabbert-Buffet N, Douvier S, Huchon C, Paganelli E, Derrien J. Role of medical treatment for symptomatic leiomyoma management in premenopausal women. J Gynecol Obstet Biol Reprod (Paris). 2011;40(8):858-74.

26. Talaulikar VS, Manyonda IT. Ulipristal Acetate: a novel option for the medical management of symptomatic uterine fibroids. Adv Ther. 2012;29(8):655-63.

27. Campo S, Garcea N. Laparoscopic myomectomy in premenopausal women with and without preoperative treatment using gonadotrophin-releasing hormone analogues. Hum Reprod. 1999;14(1):44-8.

28. Vercellin P, Trespìdi L, Zaina B, Vicentini S, Stellato G, Crosignani PG. Gonadotropin-releasing hormone agonist treatment before abdominal myomectomy: a controlled trial. Fertil Steril. 2003;79(6): 1390-5.
29. Joffe H, White DP, Crawford SL, McCurnin KE, Economou N, Connors S, et al. Adverse effects of induced hot flashes on objectively recorded and subjectively reported sleep: results of a gonadotropin-releasing hormone agonist experimental protocol. Menopause. 2013;20(9):905-14.

30. Broit K. Drug-induced osteoporosis. Rev Prat. 2012;62(2):187-92.

31. Sagsveen M, Farmer JE, Prentice A, Breeze A. Gonadotrophinreleasing hormone analogues for endometriosis: bone mineral density. Cochrane Database Syst Rev. 2003;4, CD001297.

32. Gainer EE, Ulmann A. Pharmacological properties of CDB(VA)2914. Steroids. 2003;68:1005-11.

33. Levens ED, Potlog-Nahari C, Armstrong AY, Wesley R, Premkumar A, Blithe DL, et al. CDB-2914 for uterine leiomyomata treatment: a randomized controlled trial. Obstet Gynecol. 2008;111(5):1129-36.

34. Donnez J, Tatarchuk TF, Bouchard P, Puscasiu L, Zakharenko NF, Ivanova $\mathrm{T}$, et al. Ulipristal acetate versus placebo for fibroid treatment before surgery. N Engl J Med. 2012;366(5):409-20.

35. Donnez J, Tomaszewski J, Vázquez F, Bouchard P, Lemieszczuk B, Baró F, et al. Ulipristal acetate versus leuprolide acetate for uterine fibroids. N Engl J Med. 2012;366(5):421-32.

36. Spitz IM. Clinical utility of progesterone receptor modulators and their effect on the endometrium. Curr Opin Obstet Gynecol. 2009;21(4):318-24.

37. Donnez J, Vázquez F, Tomaszewski J, Nouri K, Bouchard P, Fauser $\mathrm{BC}$, et al. Long-term treatment of uterine fibroids with ulipristal acetate. Fertil Steril. 2014;101(6):1565-73.

38. Presse-Information: Europäische Kommission erteilt Zulassung für ESMYA Typ II Variation. 